\title{
Justificación de la violencia política: Propuesta de un modelo teórico social
}

\section{[Justification of political violence. Proposal of a theoretical social model]}

\author{
Mauro Basaure (iD \\ Universidad Andrés Bello y Centro de Estudios del Conflicto y Cohesión Social
}

\begin{abstract}
Resumen
En este artículo presento un modelo teórico de análisis del fenómeno de la justificación de la violencia política capaz de integrar cuestiones sociológicas y filosóficas, el fenómeno empírico discursivo de ofrecer justificaciones y la evaluación normativa de ellas. Se reconstruye en particular el modo racionalista instrumental de justificación de la violencia política y una serie de críticas inmanentes o internas que se le pueden realizar. Junto con ello se muestran ciertos límites del modelo de la justificación de la violencia política planteado, tematizando posibilidades que exceden el marco mismo de la lógica de la justificación y obtengo ciertas conclusiones de todo ello.
\end{abstract}

Palabras clave: modelo teórico, teoría social, compensación, racionalismo, reciprocidad.

\begin{abstract}
In this article, I propose a theoretical social model for analysis of the phenomenon of the justification of political violence. The model incorporates both sociological and philosophical perspectives, addresses the empirical discursive phenomenon of offering justifications for political violence, and supports the normative evaluation of such justifications. In particular, I reconstruct the instrumental rationalist mode of justification of political violence, as well as a series of immanent or internal criticisms thereof. In addition, I acknowledge certain limitations of the proposed model by exploring possibilities that go beyond the very framework of the logic of justification. I close by offering a number of conclusions.
\end{abstract}

Keywords: theoretical model, social theory, compensation, rationalism, reciprocity

Contacto: La comunicación sobre este artículo debe ser enviada a Mauro Basaure, email mauro.basaure@gmail.com

Financiamiento: Fondo Nacional de Desarrollo Científico y Tecnológico FONDECYT N ${ }^{\circ} 1150790$; No 1191490 y Fondo de Financiamiento de Centros de Investigación en Áreas Prioritarias de la Agencia Nacional de Investigación y Desarrollo ANID-FONDAP $\mathrm{N}^{\circ} 15130009$.

Agradecimientos: A Andrés Díaz por sus comentarios a este artículo. 


\section{INTRODUCCIÓN}

En este ensayo se presenta un modelo teórico social para el análisis del fenómeno de la justificación de la violencia política que permite establecer puentes interdisciplinarios pues puede ser referido tanto a justificaciones realizadas desde la perspectiva del involucrado en actos de violencia de este tipo, y de observadores que opinan sobre ellas -ambos objetos propios al análisis social-como a tratamientos disciplinarios de carácter evaluativo y normativo sobre las justificaciones ofertadas, terreno que es ocupado mayormente por la filosofía política y moral. El modelo propuesto diferencia la lógica global de la justificación -en cuyo centro se encuentra la noción de compensación- de la cuestión evaluativa. Una serie de distinciones analíticas generan un marco general pertinente a un análisis social complejo y teóricamente informado.

\section{LA VIOLENCIA POLÍTICA Y LA LÓGICA DE LA COMPENSACIÓN}

El concepto de justificación se encuentra dentro del marco semántico de la administración de justicia. La justificación puede ser entendida como una pretensión de validez asociada a la corrección (Habermas, 1981). La operación de justificar marca siempre una diferencia pues, de ser exitosa, reconcilia a los actos en cuestión con un cierto equilibrio asociable al reino de lo justo, en el amplio y doble sentido de ajustado y de justicia (Boltanski \& Thévenot, 1991). Se parte del axioma de que la violencia política (VP) es, en principio, mero daño (DA) sin sentido como se aprecia en la Ecuación (1). Así, la justificación es la operación orientada a rescatar la VP del mero daño para reconciliarla con el mundo del sentido.

$$
\mathrm{VP}=\mathrm{DA}
$$

Para identificar la operación se propone el concepto de compensación (Frazer \& Hutchings, 2019). Justificar es ofertar una compensación para desvincular al acto de violencia de lo injusto, para que sea considerado como algo comprensible o con sentido. No se trata del sentido mentado definido por Weber (1922|2002), sino de que los actos involucrados logren hacerle sentido a un observador y evaluador externo, es decir, que se cumplan las condiciones de éxito (Latour, 2012) para que un público imparcial y diverso pueda entenderlos como actos no meramente arbitrarios.

La reconstrucción de dicha gramática supone un trabajo empírico que aquí no desarrollo. En otros ámbitos temáticos el antecedente más relevante de tal reconstrucción se encuentra en Boltanski (1990, 1993) y Boltanski y Thévenot (1991). El aporte en este ensayo es proponer un marco global en que tal gramática pueda ser inscrita una vez reconstruida. Desde esta perspectiva, hablo aquí de hacer sentido (SEN) al observador para englobar la amplia gama de posibilidades que van desde lo justo a lo meramente comprensible.

La VP sin justificación, en principio, no tiene sentido. Es solo mediante el ofrecimiento de una 
compensación (COM) que lo adquiere y ello solo en la medida que la práctica justificatoria sea exitosa. Esto se puede expresar en la equivalencia de la Ecuación (2). La justificación de la VP supone una argumentación que fundamente la corrección de un acto de esta naturaleza (i.e., justificación es fundamentación, (Pollok, 2009).

$$
\mathrm{VP}+\mathrm{COM}=\mathrm{SEN}
$$

De esto se desprenden varias cuestiones, sin embargo, la tarea inmediata es abordar una forma posible de esta fórmula general: el modelo instrumental de justificación de la VP y aspectos clave de su crítica.

\section{EL MODELO INSTRUMENTAL}

El modelo instrumental consiste en justificar la violencia en función de una oferta de compensación que tiene la forma de un estadio positivo como resultado. La VP es concebida como un medio para alcanzar un fin determinado, expuesto como compensación (Frazer \& Hutchings, 2007; Frazer \& Hutchings, 2019). De este modo, la VP deja de ser mero daño, pues se pone al servicio de un bien mayor. Aquello que se ofrece puede ser positivo en sentido proactivo y reactivo. En el caso proactivo, la oferta de compensación es alcanzar un estadio positivo ulterior y nuevo. En el reactivo, es resguardar el presente conocido evitando una situación considerada como mal mayor.

Para alcanzar un determinado estado de cosas, ofrecido proactivamente como altamente deseable, la VP es presentada como condición necesaria y suficiente para lograrlo. La sumatoria entre lo necesariamente negativo del recurso a la violencia de una parte y lo positivo de dicho estadio ulterior y nuevo, debe ser positivo. Las consecuencias de la violencia no pueden ser más negativas que el bien alcanzado. Bajo esta formulación, la VP aparece como un costo necesario a pagar $(\mathrm{CP})$ por alcanzar un bien presentado como altamente deseable (BD). De este modo, la VP es igual al daño y al CP y la compensación es alcanzar dicho bien de modo que $\mathrm{COM}=\mathrm{BD}$. La formalización de esto se muestra en la Ecuación (3).

$$
\mathrm{CP} / \mathrm{BD}=\mathrm{SEN}
$$

Dado el carácter negativo de las consecuencias de la VP y el ofrecimiento de una compensación (en la forma de un estadio positivo ulterior), la justificación es formalmente exitosa (tiene sentido) solo si el costo a pagar se mantiene dentro de un cierto margen que no supera los beneficios (ver Ecuación 4). Es decir, los costos a pagar deben ser sustantivamente menores que los beneficios a alcanzar, pues mientras más se elevan los costos, más se pierde la racionalidad básica o el sentido elemental de la justificación.

$$
\mathrm{CP} / \mathrm{BD}=\mathrm{SEN} \leftrightarrow(\mathrm{CP}<\mathrm{BD})
$$

En el caso reactivo, el bien ofrecido como compensación es evitar un mal mayor (EMMay). Aplica la misma equivalencia, solo que la VP adquiere la forma de un mal menor (MMen), donde VP = DA = MMen. Ello permite, en principio, inscribir la VP en el marco del sentido, como muestra la Ecuación (5). 


$$
\text { MMen/EMMay }=\text { SEN }
$$

Para ello, igual que en la Ecuación (4), debe cumplirse que el daño producido por la VP sea menor que el mal que busca evitarse (ver Ecuación 6). De no cumplirse esta condición se pierde también la racionalidad básica de la justificación. Tanto como costo a pagar por un bien o como mal menor respecto de uno mayor, se cumple el axioma del modelo: la VP es un mal. Lo que cambia es el tipo de oferta.

$$
\frac{\text { MMen }}{\text { EMMay }}=\text { SEN } \leftrightarrow(\text { MMen })<(\text { EMMay })
$$

Estos ejemplos muestran una lógica instrumental $\mathrm{y}$ racional, que ha sido fuertemente puesta en cuestión. Este modelo permite ver una dimensión anexa a la lógica de la compensación, que hace parte de la formula general de la justificación: el diferencial entre daño y beneficio. Consiste en generar la mayor diferencia posible entre el daño asociado a la VP y el valor positivo de la compensación. Ese diferencial se puede generar de dos maneras: ampliando el beneficio de la compensación ofrecida o disminuyendo los daños de la VP. El mayor diferencial posible se genera combinando ambas formas. En la lógica proactiva y la reactiva, la devaluación del daño solo acentúa el diferencial de valor ( $\mathrm{VP}<\mathrm{COM})$, que es parte clave de las condiciones de sentido de la justificación. Cuando una instancia evaluativa estima la justificación de la VP, esa estimación puede referirse tanto a la devaluación de los daños, como a la justificacióncompensación ofertada, o ambas a la vez; es decir, a la fórmula justificatoria en su conjunto.

\section{LAS CRÍTICAS DEL MODELO INSTRUMENTAL}

El modelo instrumental puede ser objeto de varias críticas desde la perspectiva normativa que conducen al rechazo absoluto o relativo de las ofertas de justificación, según las categorías expuestas previamente. Esas críticas competen típicamente a la filosofía política y moral, pero más allá de ésta-es posible reconstruir una crítica inmanente que ataca los supuestos sobre los que se erige la justificación instrumental. El lugar analítico de esta crítica no es el momento evaluativo desde la perspectiva del observador externo, sino uno interno, basado en un análisis crítico apoyado en la tradición de la teoría social.
No se trata, por tanto, de críticas basadas en categorías filosófico-morales, sino que son inmanentes en el sentido de que se realizan en función de los problemas que se derivan de la estructura misma del modelo de justificación. Para realizar esa reconstrucción crítica es necesario y útil traducir la fórmula de justificación (en su determinación instrumental) en términos de teoría de la acción. Consecuente con lo anterior, se identifican tres críticas inmanentes al modo instrumental de justificación, íntimamente relacionadas entre sí, pero analíticamente diferenciables. 
Sesgo objetivista de la justificación instrumental

Hay una crítica del acto unidad -en cuyo marco Parsons (1937|1968) desarrolló la teoría voluntarista de la acción- extrapolable a la formulación instrumental de la justificación de la VP, que identifica un sesgo objetivista que conduce a no reconocer las limitaciones de las capacidades cognitivas de los actores o sus debilidades y las determinaciones subjetivas sobre la definición y manejo de la situación de la acción (Joas, 1996; Joas \& Knöbl, 2004).

Sesgo objetivista significa que el modelo de la acción asume erróneamente que los actores tienen la capacidad perfecta de evaluar y definir los medios adecuados, y reconocer las condiciones de la acción objetivamente. Frente a esto, es correcto revindicar la relevancia que juega el componente subjetivo. El conocimiento de la situación de la acción y la definición de los medios están típicamente intervenidos por la subjetividad, por lo que no pueden ser determinadas desde el exterior, sino que deben considerarse las capacidades interpretativas de los actores, pues en función de ellas se definen las situaciones. El teorema de Thomas y Thomas (1928|1938) valida esta idea que es reforzada por la tradición de la etnometodología y el interaccionismo simbólico (Sanderson, 2001).

Esta crítica se aplica a toda justificación de la violencia. La justificación opera haciendo una descripción idealizada de la relación entre medios y fines como puramente objetiva, no solo idealizando un estado final -proactivo o reactivocuya realización es presentada como necesaria, sino que también se asegura que el único medio a disposición para alcanzarlo es la violencia. La VP aparece como condición necesaria y suficiente para alcanzar el fin propuesto como compensación. No debe ni puede existir ninguna alternativa, pues de otro modo se cae la justificación. Esto muestra las dos alternativas en juego: una sincrónica y una diacrónica o dinámica. La sincrónica supone una especie de cálculo lógico matemático según el que para un fin cualquiera hay solo un medio adecuado. Para la alternativa diacrónica se admiten varios medios posibles donde la VP es el último en una serie donde todos los medios pacíficos anteriores ya fueron probados de manera exhaustiva sin el resultado esperado.

Las condiciones objetivas de una justificación instrumental de la VP son complejas, pero incluso si pudiesen cumplirse, no significa que pueda aceptarse sin más como justificación. Esa descripción objetiva de relación entre medios y fines ignora la dimensión subjetiva, según la cual en toda acción y definición de la situación operan las capacidades interpretativas de los actores que no son ajenas a la influencia de discursos y relaciones de poder. Hacer pasar lo subjetivo por una relación puramente objetiva, supone una especie de contrabando en la justificación instrumentalista de la cual toda instancia o momento evaluativo debe estar consciente.

Consideración de las consecuencias de la acción

Directamente relacionada con lo anterior está la crítica inmanente según la que el modelo de la acción definida en términos de la prosecución de fines o realización de valores excluye las 
consecuencias de la acción. Esta crítica recuerda que la acción no termina con el logro de los fines. El modelo de Parsons (1937|1968) del acto unidad es puesto en cuestión como un modelo que analiza la acción en aislamiento. Eso significa que no se considera el encadenamiento e interdependencia de las acciones de varios actores y las consecuencias de ellas, ni el hecho de que las consecuencias de las acciones tienen repercusiones sobre el propio actor, pudiendo transformar sus propósitos y poniéndolo en situación de tener que reaccionar a las consecuencias de sus propias acciones (Joas \& Knöbl, 2004).

Es Merton (1949 | 1957) quien planteó la diferencia entre las consecuencias intencionales $y$ no intencionales de la acción. Esa distinción desdobla el campo cognitivo entre aquello subjetivamente buscado y aquello objetivamente ocurrido, lo que remite a las limitaciones de la perspectiva del actor. Los actores pueden, por ejemplo, saber de las consecuencias no intencionales indeseables de la acción e introducirlas en el cálculo. De ahí se deriva la relevancia de la distinción entre consecuencias previstas y no previstas o anticipadas de la acción y se genera también un juego de posibilidades entre consecuencias intencionales y no intencionales, deseables e indeseables. En este argumento son relevantes aquellas no intencionales, indeseables y no anticipadas (Boudon, 1982), pues la compleja interdependencia de la vida moderna significa que el campo de las consecuencias no previstas es mucho mayor que el de aquellas previstas.

Esta problemática puede extrapolarse como crítica inmanente de la justificación instrumental de la VP. Según el modelo propuesto, la justificación supone que su oferente brinde una garantía del cumplimiento de la condición de que el daño producido por la VP se mantenga bajo cierto rango esperable (caso proactivo) o sea menor que el mal que busca evitarse, como en el caso reactivo. (ver Ecuaciones 4 y 6). Las consecuencias de la VP pueden considerarse de dos maneras. Dentro de la fórmula de la justificación, los efectos de la violencia no deben ser intencionales (i.e., un fin en sí mismo). Se trata de una intencionalidad de medios, es decir, del buen manejo racional de un recurso que, desde el punto de vista del modelo, se entiende como un daño (ver Ecuación 1).

A ello puede sumarse dentro del cálculo la existencia de efectos no intencionales previstos, deseables y no, también llamados colaterales, pero sigue existiendo un campo de efectos no intencionales, imprevistos, indeseables, que no son controlables por el actor. No se puede asegurar que la VP, como costo a pagar por alcanzar un bien, se mantenga bajo cierto rango menor, o que como mal menor sus consecuencias negativas no superen el mal mayor que se quiere evitar. Por lo mismo, dada esta incertidumbre sobre los resultados, en estos casos el oferente recurrirá a la devaluación de las consecuencias de la VP o el ensalzamiento del fin que se persigue con ella, enfatizando una certeza ficticia que oculta dicha incertidumbre.

Dependencia de la justificación del logro de los fines propuestos

Cuando la VP es concebida como medio, su justificación depende de la realización de la compensación ofertada. En los dos puntos anteriores se ha dado por sentado que los fines 
se consiguen y con ello se logra establecer las condiciones internas de justificación. En muchas situaciones empíricas ese supuesto no se cumple y el logro del fin se prolonga por un tiempo indefinido y con ello se dilata la VP y sus consecuencias. Esto muestra que hay una condición de validez básica de la justificación instrumental, que es de carácter temporal. Desde el punto de vista de esta validez, el recurso a la VP funciona contra reloj, pues mientras más se dilata la consecución del fin compensatorio, la justificación instrumental de la VP pierde más poder persuasivo.

La Ecuación (3) supone que la consecución del $\mathrm{BD}$ sea algo inminente o que no se prolongue demasiado. En la Ecuación (5), el mal mayor es pura potencialidad. Las condiciones de éxito son que EMMay no tenga lugar. La urgencia aquí está dada porque, de no recurrir a la VP, el mal mayor ocurrirá. La intervención violenta también debe ser lo más rápida y efectiva posible, y no debe prolongarse en el tiempo pues, de otro modo, se hace cada vez menos plausible que el mal menor sea efectivamente menor que el mal mayor. En ambos casos está comprometido el sentido de la justificación. Esas condiciones operan como elementos críticos inmanentes y como criterios de evaluación frente a situaciones empíricas en que se pretende dar carta blanca a la VP en función del logro de un fin proactivo o reactivo.

Otro aspecto del cual es imprescindible estar consciente, es que la justificación instrumental de la VP supone una doble lógica temporal ex ante y ex post. Por un lado, la justificación tiene la forma de una oferta o promesa doble: no se trata solo de conseguir el fin (proactivo o reactivo) sino de hacerlo cumpliendo con la condición de que $\mathrm{CP}$ sea menor que $\mathrm{BD}$, o que MMen sea menor que el mal a evitar. Esa promesa significa un compromiso verbal o simbólico, y de ahí una forma de obligación social que busca ser garantía de cumplimiento. Pese a ello, solo el cumplimiento real del doble compromiso asegura el sentido de la justificación. Una justificación ex ante es necesaria, pero no suficiente pues resulta puramente subjetiva si no alcanza el momento objetivo de hacerse real. El que las consecuencias devengan algo distinto de lo intencionado en la acción abre al conflicto trágico entre lo subjetivo (la acción) y lo objetivo (el hecho), entre lo espiritual y lo natural (Basaure, 2006).

Como se sigue del modelo propuesto, lo anterior supone la necesidad lógica de una instancia evaluativa respecto del cumplimiento (oportuno) de la promesa en las dos dimensiones temporales mencionadas. Esta instancia debe ser diferenciada del momento evaluativo, tematizado más arriba. No se trata aquí de la cuestión normativa y externa (sobre el carácter absoluto, relativo o condicional de la justificación de la VP), sino de la cuestión (funcional, interna y relativa a la eficacia) de si se cumple o no el compromiso que supone dicha justificación. Esta doble lógica temporal y su dependencia de la eficacia ex post, no significa únicamente mostrar una característica analítica propia de este fenómeno, sino fundamentalmente realizar una crítica inmanente. Toda justificación instrumental de la VP, en su estado de compromiso, es incompleta o subjetiva, debe considerarse improbable y en 
ningún caso garantiza el logro de su aspecto objetivo complementario. La justificación de la VP supone una afirmación subjetiva que, siguiendo a Hyppolite (1953), hace como si conociera integralmente todas las circunstancias de la acción.

\section{MÁS ALLÁ DEL RACIONALISMO DE LA JUSTIFICACIÓN}

Con ayuda de la teoría social, en las páginas precedentes se han identificado algunas críticas inmanentes al modelo instrumental. Hay otro tipo de críticas a toda concepción de la acción social (sea utilitarista o que considere el cumplimiento de normas) en términos del esquema racionalista medio-fin. Esa crítica abre espacio a aquellas formas de acción que no se enmarcan en dicho esquema. Es el caso de acciones rutinarias que se realizan de modo preconsciente y que requieren de un esfuerzo para ser puestas a disposición de la conciencia (Cohen, 1989; Giddens, 1991|2013). Como la VP es terreno de lo extraordinario, es difícilmente tratable desde este ángulo.

Distinto es el caso de aquellas acciones de autoexpresión, que no se conciben bajo una lógica racionalista porque el actor no persigue un fin con su acción, no se guía por normas colectivas, ni pueden entenderse como planeadas racionalmente para alcanzar un objetivo que reside fuera de la acción misma (Joas, 1996; Joas \& Knöbl, 2004; Jonas \& Littig, 2016). Cuando se trata de autoexpresión, el sentido de la acción se revela al actor en la misma realización de la acción y gracias a esa realización. La VP puede ser tematizada desde este ángulo no racionalista. Con la ayuda del modelo general presentado, se pueden ahora analizar los vínculos entre la VP y el sentido, que no pasan por dicho modelo ni por la lógica de la oferta de una compensación.
Estetización de la violencia política

Tomando como referencia el modelo general de justificación se puede identificar conceptualmente un discurso sobre la VP que hace añicos la fórmula de la justificación. Me refiero a cuando la VP no es concebida como daño sino como un bien o experiencia de disfrute. Con esto, no se está ya en la lógica de la justificación, sino en la de la apología. Esto significa que el momento evaluativo ya no es considerado por el actor participante quien se desentiende de ofrecer una compensación. La VP tratada como un bien de goce estético, afectivo, adrenalínico y vertiginoso (Huët, 2019), solo puede ser una cuestión personal que abdica de una evaluación y apelación externa. Este caso puede concebirse como negación del axioma del modelo propuesto y debe ser tematizado.

No se trata aquí de la estética de la violencia, que puede encontrarse en el arte, sino de la VP como acto autoexpresivo que puede o no contar con un discurso explícito. Cuando no es el caso, se escapa aún más de la fórmula de la justificación, pues no solo implica la negación de su axioma, sino también el adentrarse en el terreno del análisis de las motivaciones ocultas de parte de un tercero (e.g., un psicoanalista, etnógrafo, antropólogo). En el contexto de esta propuesta, es relevante esta dimensión en la medida que permite ver 
indirectamente o desde afuera que el análisis racionalista de la justificación de la VP en términos utilitaristas o normativos tiene límites y su uso (auto-justificativo o de imputación externa) puede cubrir con un manto racionalista acciones cuya estimulación reside en otro lugar, más allá de la lógica de la justificación.

Expresión pre-política de la violencia política

Un segundo caso en que la noción de autoexpresión juega un rol es el que se puede asociar con los disturbios más o menos espontáneos. En ellos normalmente no se encontrará una oferta de compensación. Al igual que en el caso anterior, la falta de discurso explícito es generalmente suplida por una oferta externa de imputación sobre motivaciones. En este contexto se recurre típicamente a nociones como la de violencia estructural (Galtung, 1969).

La VP sería una de las formas posibles de expresión de dicha violencia primera, de carácter sistémico o estructural. De esto se derivan dos cuestiones relevantes. Primero, es necesario entender este proceso como dinámico. Ello significa que, dentro del proceso de articulación política se articulen también, por ejemplo, discursos de justificación bajo un esquema racionalista. Ello es plausible si se entiende que las luchas sociales viven procesos de objetivación en los que los procesos de articulación discursiva son normales (Boltanski, 1982). Las formas particulares y concretas de justificación no nacen de la nada. Lo que nace en un ámbito, que puede denominarse pre-político, puede devenir político (Honneth, 2010). En segundo lugar, la oferta explicativa de violencia estructural (que aquí reconstruye las fuentes motivacionales de la VP y las encuentra en un ámbito pre-político) introduce un elemento nuevo: la lógica de una reciprocidad negativa (Jacoby, 2007). La explicación de carácter sociológico (no necesariamente disciplinar) opera aquí como productora de sentido. No hay compensación sino explicación (EX) como muestra la Ecuación (7). Para muchos, puede tener sentido que la sociedad sufra las consecuencias de una violencia pre-política a cambio de la violencia estructural que ella ha ejercido sobre algunos de sus miembros.

$$
\mathrm{VP} / \mathrm{EX}=\mathrm{SEN}
$$

Ese sentido no significa que la VP pierda el carácter subjetivo (arbitrario, monstruoso o alienígena en el sentido de una singularidad no reconocible). Solo significa que logra ser inscrita dentro de un relato comprensible de reciprocidad, aunque sea negativa. No significa acuerdo, ni menos apoyo a esa VP. Se trata de una modalidad que escapa a la visión racionalista, en el sentido de que esa VP es, en principio, pura autoexpresión y no puede inscribirse dentro de un esquema medio-fin pues no persigue nada, ni utilitaria, ni normativamente.

\section{CONCLUSIONES}

El aporte en teoría social no siempre consiste en responder preguntas de investigación sino en establecer los términos en que debe plantearse dicha pregunta. En este ensayo no se respondió a la pregunta por la justificación de la VP, sino que se propuso un modelo para analizar esa pregunta y estructurar distintas perspectivas. 
Pese a ello, se pueden obtener dos conclusiones sustantivas: (a) Desde la crítica inmanente al modelo racionalista: toda justificación de la violencia en sentido de una oferta instrumental supone un nivel bajo de reflexividad y un nivel alto en voluntarismo subjetivo, el sesgo objetivista y la abstracción de las consecuencias objetivas hacen que el tipo de justificación se afirme en supuestos y certidumbres sostenibles más con voluntad y convicción que con reflexividad. Esta última introduce elementos de incerteza y ambivalencia que le resultan ciertamente incompatibles. (b) La teoría e investigación social haría bien en explorar el fenómeno de la VP más allá de la lógica y el sentido de la justificación, pues bajo un modelo más amplio de acción, es posible integrar dimensiones del sentido que parecen colmar los hechos de VP. No se trata aquí de la dicotomía racional e irracional, sino de los límites de una lógica racionalista y justificatoria para comprender determinados hechos sociales. Es posible que el tipo de acción afectiva juegue un rol igual o más importante que el tipo de acción con arreglo a fines o a valores de Weber (1922 | 2002).

\section{REFERENCIAS}

Basaure, M. (2006). El Edipo y las bases de una sociología del hecho en la estética hegeliana. Ciencias Sociales Online, 3(2), 1-26. Recuperado de

https://web.archive.org/web/2015051312231 6/http://www.uvm.cl/csonline/2006_2/pdf/ba saure.pdf

Boltanski, L. (1982). Les cadres: La formation d'un groupe social. Paris, Francia: Les Editions de Minuit.

Boltanski, L. (1990). L'amour et la justice comme compétences: Trois essais de sociologie de l'action. Paris, Francia: Editions Métailié.

Boltanski, L. (1993). La souffrance à distance: Morale humanitaire, médias et politique. París, Francia: Editions Métailié.

Boltanski, L., \& Thévenot, L. (1991). De la justification: Les économies de la grandeur. Paris, Francia: Éditions Gallimard.
Boudon, R. (Ed.). (1982). The unintended consequences of social action ( $1^{\text {a }}$ ed.). London, UK: Palgrave Macmillan.

Cohen, I. (1989). Structuration theory: Anthony Giddens and the constitution of social life. London, UK: Palgrave Macmillan.

Frazer, E., \& Hutchings, K. (2007). Argument and rhetoric in the justification of political violence. European Journal of Political Theory, 6, 180-199. doi: 10.1177/1474885107074349

Frazer, E, \& Hutchings, K. (2019). Can political violence ever be justified? Cambridge, UK: Polity Press.

Galtung, J. (1969). Violence, peace, and peace research. Journal of Peace Research, 6, 167-191. doi: 10.1177/002234336900600301

Giddens, A. (1991| 2013). The constitution of society: Outline of the theory of structuration (Kindle ed., Vol. 1). Cambridge, UK: Polity Press. 
Habermas. (1981). Theorie des kommunikativen handelns, band 2: Zur kritik der funktionalistischen vernunft. Frankfurt am Main: Suhrkamp Verlag.

Honneth, A. (2010). Kampf um anerkennung: Zur moralischen grammatik sozialer konflikte (6 a ed.). Frankfurt am Main: Suhrkamp Verlag.

Huët, R. (2019). Le vertige de l'émeute: De la zad aux gilets jaunes. Paris, Francia: Presses Universitaires de France.

Hyppolite, J. (1953). Logique et existence: Essai sur la logique de Hegel. Paris, Francia: Presses universitaires de France.

Jacoby, T. (2007). Understanding conflict and violence: Theoretical and interdisciplinary approaches. New York, NY: Routledge.

Joas, H. (1996). Die kreativität des handelns. Frankfurt am Main: Suhrkamp Verlag.

Joas, H., \& Knöbl, W. (2004). Sozialtheorie: Zwanzig einführende vorlesungen. Frankfurt am Main: Suhrkamp Verlag.

Jonas, M., \& Littig, B. (2016). Praxeological political analysis. London, UK: Routledge.

Latour, B. (2012). Enquête sur les modes d'existence. Paris, Francia: Éditions La Découverte.
Merton, R. K. (1949|1957). Social theory and social structure (Rev. ed.). Glencoe, IL: The Free Press.

Parsons, T. (1937|1968). The structure of social action (Volumen II: Weber): A study in social theory with special reference to a group of recent European writers (1 ${ }^{\text {a }}$ Press Paperback ed.). New York, NY: The Free Press.

Pollok, K. (2009). Begründen und rechtfertigen: Eine untersuchung zum verhältnis zwischen rationalen erfordernissen und prävalenten handlungsgründen. Berlin, Alemania: De Gruyter.

Sanderson, S. K. (2001). The evolution of human sociality: A darwinian conflict perspective. Lanham, MD: Rowman \& Littlefield Publishers.

Thomas, W. I., \& Thomas, D. S. (1928|1938). The child in America: Behavior problems and programs. New York, NY: Knopf.

Weber, M. (1922|2002). Wirtschaft und gesellschaft: Grundriss der verstehenden soziologie ( $5^{\mathrm{a}}$ ed.). Tübingen: Mohr Siebeck.

Manuscrito recibido: 16-03-2020 Manuscrito aceptado: 15-06-2020 